\title{
Soluções Envoltórias para EDPs com Dois Conjuntos Disjuntos de Variáveis
}

\author{
Maria Lewtchuk Espindola, \\ Depto de Matemática, DM/CCEN, UFPB, \\ 58051-970, João Pessoa, PB \\ E-mail: mariia@mat.ufpb.br.
}

$28 / 09 / 2013$

Resumo: Soluções envoltórias no caso das EDPs, hipersuperfícies que envolvem uma das famílias de hipersuperfícies dadas pelas soluções completas, tem inúmeras aplicações. O intuito deste artigo é desenvolver e discutir a existência de soluções envoltórias de EDPs que envolvem dois conjuntos disjuntos de variáveis, como é o caso das variáveis canônicas do espaço de fase na Mecânica Analítica Hamiltoniana.

Palavras-chave: Soluções envoltórias, Mecânica Analítica Hamiltoniana, Procedimento de Hamiltonização alternativa, Espaço de fase, Matemática aplicada. 


\section{Introdução}

O papel desempenhado pelas soluções envoltórias em EDOs e EDPs é muito importante, principalmente em matemática aplicada. No caso das EDPs as soluções envoltórias são as hipersuperfícies que envolvem uma das famílias de hipersuperfícies dadas pelas soluções completas. O intuito desse artigo é desenvolver e discutir as soluções envoltórias de EDPs que envolvem dois conjuntos disjuntos de variáveis. Esse tipo de EDPs aparecem na Mecânica Hamiltoniana, desde que o espaço de fase é composto por dois conjuntos disjuntos de variáveis canônicas: as coordenadas e os momenta generalizados, veja, por exemplo, Leech ou Goldstein [4]. No procedimento de Hamiltonização alternativa mostramos que o Hamiltoniano é definido por uma EDP e que a função Hamiltoniana clássica é a solução envoltória da solução linear nos momenta [2]. Como a técnica de determinação das envoltórias foi utilizada sem demonstração no procedimento de Hamiltonização a intenção desse artigo é fundamentar o que foi utilizado estendendo o procedimento de determinação das soluções envoltórias para o caso do espaço de fase e demonstrando que em determinados casos essa solução não existe.

\section{Soluções Envoltórias para Funções que Depen- dem de Dois Conjuntos Disjuntos de Variáveis}

Como temos interesse na aplicação de soluções envoltórias na Mecânica Hamiltoniana e o espaço de fase é composto por dois conjuntos de variáveis, vamos considerar esses conjuntos de variáveis como $x=x_{1}, \ldots, x_{n}$ e $y=$ $y_{1}, \ldots, y_{n}$ e a função $u=u(x, y)$. A EDP fica dada por

$$
u(x, y)=f(p, q, x, y)
$$

onde $p=p_{1}, \ldots, p_{n}, \quad p=\partial u / \partial x$ e $q=q_{1}, \ldots, q_{n}, \quad q=\partial u / \partial y$. Escrevendo as soluções gerais como

$$
\varphi(u, x, y)=0,
$$

obtemos uma família de soluções completas como

$$
\varphi(u, x, y, a, b)=0,
$$

onde $a=a_{1}, \ldots, a_{n}$ e $b=b_{1}, \ldots, b_{n}$ são constantes. Impondo as condições de envoltória [5], obtemos

$$
\frac{\partial \varphi}{\partial a_{i}}=0, \quad \frac{\partial \varphi}{\partial b_{i}}=0
$$


Então a solução do sistema de equações (3) e (4) determina a solução envoltória.

Vamos supor que podemos explicitar a solução geral

$$
u(x, y)=\phi(x, y),
$$

logo desta temos a solução completa

$$
u(x, y)=\phi(x, y, a, b) .
$$

As condições de envoltória equações (4) são

$$
\frac{\partial \phi}{\partial a_{i}}=0, \quad \frac{\partial \phi}{\partial b_{i}}=0 .
$$

Sendo que este sistema de $2 n$ equações determina $a$ e $b$ e portanto a solução envoltória de (6).

Se, por outro lado, a equação (1) for linear em $p$ (ou $q$ ) e não depender $q$ (ou de $p$ ) então esta equação não possui solução envoltória. Por exemplo, considere a EDP

$$
u(x, y)=\left(y_{i}-r_{i}(x)\right) q_{i}+s(x),
$$

${ }^{1}$ cuja solução geral, se $y_{i} \neq r_{i}$, será

$$
u=s+\psi_{i}\left(y_{i}-r_{i}\right),
$$

onde $\psi_{i}=\psi_{i}(c)$ e $c=c_{1}, \ldots, c_{n}$.

Considerando agora a solução completa equivalente a (3)

$$
\varphi=s+a_{i}\left(y_{i}-r_{i}\right)-u=0,
$$

onde $a_{i}$ são constantes arbitrárias, a imposição da condições de envoltória - equação (7), resulta em $y_{i}-r_{i}=0$, contrariando a hipótese anterior. Portanto no caso especificado a equação diferencial parcial não possui envoltória.

\section{Soluções envoltórias no procedimento de Hamil- tonização alternativa}

\subsection{Hamiltonização}

Definimos Hamiltonização, para sistemas mecânicos com $N$ graus de liberdade descritos por uma função Lagrangiana, como o procedimento dado pelos seguintes passos:

\footnotetext{
${ }^{1}$ Índices repetidos implicam em soma.
} 
i. Existe uma função chamada de Lagrangiano do sistema $L(q, \dot{q}, t)$ que descreve este através das equações de Euler-Lagrange, i.e.,

$$
\frac{d}{d t}\left(\frac{\partial L}{\partial \dot{q}_{i}}\right)-\frac{\partial L}{\partial q_{i}}=0
$$

ii. É possível definir um novo conjunto de variáveis independentes $p_{i}$ (denominadas de momenta do sistema) e uma variável dependente $H(p, q, t)$ chamada de Hamiltoniano do sistema, tal que exista a seguinte relação entre as novas variáveis e as anteriores

$$
H(p, q, t) \equiv p_{i} \dot{q}_{i}-L(q, \dot{q}, t)
$$

iii. O movimento do sistema mecânico pode ser descrito pelas equações (11), ou de forma alternativa, pelo conjunto de equações canônicas de movimento, conhecidas como equações de Hamilton:

$$
\begin{aligned}
\dot{q}_{i} & =\frac{\partial H}{\partial p_{i}} \\
\dot{p}_{i} & =-\frac{\partial H}{\partial q_{i}} .
\end{aligned}
$$

A equivalência entre as descrições Lagrangiana (i) e Hamiltoniana, definida pelos passos (ii) e (iii), é obtida sempre que:

$$
\frac{d}{d t}\left[\frac{\partial L}{\partial\left(\partial H / \partial p_{i}\right)}\right]-\frac{\partial L}{\partial q_{i}}=0
$$

onde o Lagrangiano é considerado como função de $q_{k}, \partial H / \partial p_{k}$ e $t$. [4]

\subsection{Procedimento De Hamiltonização Alternativa}

O procedimento de Hamiltonização consiste na substituição do primeiro conjunto de equações de Hamilton - (13), na definição da função Hamiltoniana - (12) resultando em

$$
H=p_{i} \frac{\partial H}{\partial p_{i}}-L\left(q, \frac{\partial H}{\partial p}, t\right),
$$

que passa a ser a equação diferencial parcial que define a função Hamiltoniana. Portanto, qualquer solução desta equação será uma descrição canônica do sistema mecânico. 
Esta equação diferencial parcial possui uma solução generalizada linear nos momenta dada por

$$
H=p_{i} A_{i}-L(q, A, t)
$$

onde os $A_{i}{ }^{\prime} \mathrm{s}$ são funções arbitrárias de $q_{k}$ e $t$, que serão determinadas a partir do primeiro conjunto de equações de Hamilton (13) e pelas relações de equivalência entre as descrições Hamiltoniana e Lagrangiana (15)..

Por outro lado, se o Lagrangiano não é singular, então a equação (16) possui também uma solução envoltória (singular), que é obtida através das seguintes condições:

$$
\frac{\partial H}{\partial A_{i}}=0
$$

Estas condições de envoltória determinam as funções arbitrárias $A_{i}$.

Portanto, podemos concluir que se $L$ é uma função Lagrangiana não singular (regular) teremos duas maneiras alternativas de Hamiltonizar o sistema mecânico: (a) o Hamiltoniano é uma solução particular obtida de (17); (b) o Hamiltoniano é a solução envoltória de (16).

É importante ressaltar que, em todo o procedimento desenvolvido, não foi utilizada a definição usual dos momenta $(p=\partial L / \partial \dot{q})$. Efetivamente os momenta passam a ser definidos pelo segundo conjunto de equações de movimento de Hamilton - (14).

Se escolhemos a alternativa (a) para Hamiltonizar o sistema os momenta obtidos a partir das equações (14) diferem daqueles obtidos a partir da definição usual. Enquanto que, se a escolha for a alternativa (b) para a Hamiltonização do sistema, então podemos demonstrar que as equações (14) conduzem as definições usuais dos momenta e portanto a hamiltonização usual.

A partir de (18) e (17) temos

$$
\frac{\partial H}{\partial A_{i}}=p_{i}-\frac{\partial L}{\partial A_{i}}=0 .
$$

Substituindo (20) em (19) e utilizando as equações de Euler-Lagrange - (11), obtemos

$$
\dot{p}_{i}=\frac{\partial L}{\partial q_{i}}=\frac{d}{d t}\left(\frac{\partial L}{\partial \dot{q}_{i}}\right),
$$

as quais reproduzem as definições usuais dos momenta.

A conclusão é que este procedimento de Hamiltonização contém o usual sempre que este existe e define uma nova Hamiltonização em qualquer caso, inclusive naqueles em que o procedimento usual não nos fornece quaisquer resultados. [2] 


\section{Conclusão Final}

A demonstração da inexistência em determinados casos da solução envoltória é o que fundamenta a necessidade de uma abordagem que difere da de Hamilton para os sistemas singulares como, por exemplo, a desenvolvida por Dirac [1] e por Espindola [2]. Este artigo poderia ser generalizado estendendo a um número $\mathrm{n}$ de conjuntos disjuntos de variáveis. Assim como para o caso continuo envolvendo portanto EDPs variacionais. No procedimento de Hamiltonização alternativa que desenvolvemos para teorias de campo utilizamos esses conceitos [3].

\section{Referências}

[1] Dirac, P. A. M., Generalized Hamiltonian Dynamics, Can. J. Math., vol. 2, pp. 129-148, 1950.

[2] Espindola, M. L., Espindola, O. e Teixeira, N. L. - Hamiltonization as a Two Fold Procedure, Hadronic J., vol. 28, pp. 807-810, 1986; Espindola, M. L., Espindola, O. e Teixeira, N. L. Hamiltonization for Singular and Non Singular Mechanics, J. Math. Phys., vol. 9, pp. 121-124, 1986; Espindola, M. L., Espindola, O. e Teixeira, N. L. - Direct Hamiltonization, Hadronic J. Suppl., vol. 4, pp. 369-373, 1996; Espindola, M. L. - Hamiltonizações Alternativas, Tese de Doutorado, DF UFRJ, 1993; Espindola, M, L., Direct Hamiltonization - Generalization Of An Alternative Hamiltonization, Int. J. Bifurcation Chaos, vol. 22, pp. 1250135 [5 pages ], DOI No: 10.1142/S0218127412501350, (2012).

[3] Espindola, M. L., Espindola, O. e Teixeira, N. L. - Two Fold Hamiltonization for Field Theory, Hadronic J., vol. 10, pp. 83-87, 1987.

[4] Leech, J. W., "Mecânica Analítica", Ao Livro Técnico, EDUSP, RJ, 1971; Goldstein, H., "Classical Mechanics", Addison Wesley, 1971.

[5] Sneddon, I., Elements of Partial Differential Equations, MCGRAWHILL, Kogakusha, First edition, 1957. 